\title{
Lenguaje y Teoria de la Mente: una aproximación multidimensional
}

\author{
Mariela Resches ${ }^{1}$, Elisabet Serrat ${ }^{2}$, Carles Rostan ${ }^{2}$ \\ Y MOISÉS ESTEBAN ${ }^{2}$
}

${ }^{1}$ Universidade de Santiago de Compostela; ${ }^{2}$ Universitat de Girona

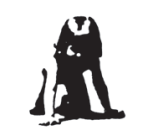

Resumen

El propósito de este trabajo es realizar una puesta al día sobre las relaciones entre el desarrollo del lenguaje y el desarrollo de la Teoría de la Mente. Entre las hipótesis propuestas para conceptualizar dichas relaciones, realizamos un examen más exhaustivo de aquellos modelos que apoyan una implicación directa entre lenguaje y Teoría de la Mente. En este contexto, en primer lugar subrayamos la necesidad de ampliar dicha noción para incluir habilidades anteriores y posteriores a la comprensión de la falsa creencia. En segundo lugar, examinamos las diferentes hipótesis acerca de los aspectos del lenguaje más vinculados al desarrollo sociocognitivo, y las principales evidencias empíricas que las sustentan. Finalmente, discutimos un modelo de causalidad reciproca donde los vínculos entre lenguaje y Teoría de la Mente variarán según el momento del desarrollo considerado.

Palabras clave: Teoría de la Mente, desarrollo del lenguaje, comunicación, desarrollo Sintáctico, comprensión social.

\section{Language and Theory of Mind: A multidimensional approach}

\begin{abstract}
The paper aims to update the relationships between language development and Theory of Mind. Through the hypotheses put forth to conceptualise these relationships, we undertake an in-depth assessment of those models that support a direct implication between language and Theory of Mind. In this context, first we underline the need to expand our view of Theory of Mind in order to include a wider range of sociocognitive abilities, acquired before and after false belief understanding. Second, we examine different hypotheses on language aspects closely linked to sociocognitive development and the main empirical evidence supporting them. Finally, we discuss a reciprocal causality model in which the links between language and Theory of Mind change depending to the stage of development being considered.

Keywords: Theory of Mind, language development, communication, syntactic development, social understanding.
\end{abstract}

\footnotetext{
Agradecimientos: Mariela Resches desea agradecer la ayuda concedida por la Dirección Xeral de Investigación, Xunta de Galicia, para la realización de una estancia de investigación en la Universitat de Girona. La redacción de este artículo se ha elaborado al amparo del proyecto SEJ2006-12039/PSIC.

Los autores agradecen los comentarios y sugerencias de dos revisores anónimos, que han contribuido a mejorar sustancialmente el manuscrito original

Correspondencia con los autores: Mariela Resches. Departamento de Psicología Evolutiva y de la Educación. Universidad de Santiago de Compostela.15782 Santiago de Compostela. E-mail: mariela.resches@usc.es
} 


\section{Introducción}

Originalmente importado de la primatología (Premack y Woodruff, 1978), Teoría de la Mente (en adelante, TM) es un término que refiere a la capacidad que permite a los sujetos explicar la propia conducta y la de los demás basándose en la atribución de estados mentales (Carpendale y Lewis, 2006; Perner, 1991; Wimmer y Perner, 1983).

En sus comienzos, la investigación en TM encontró en la comprensión de la falsa creencia su más fructífera definición operacional. De acuerdo al consenso implícito entre los autores, si entendemos que la mente es un sistema representacional, la emergencia de una TM debería identificarse con un salto evolutivo que se expresa en la comprensión de la falsa creencia, entre los cuatro y los cinco años de edad. Poco a poco, sin embargo, autores con una importante tradición de investigación en el área como Judy Dunn (Dunn, 1988), Henry Wellman (Wellman, 1993) o Claire Hughes (de Rosnay y Hughes, 2006), entre otros, comenzaron a objetar este enfoque restringido para adoptar una visión más gradualista del problema. Esto implica ampliar la noción de TM para pasar a considerar que se trata de una serie de adquisiciones conceptuales cada vez más elaboradas y flexibles para explicar la conducta humana. Bajo esta nueva perspectiva, algunos autores han adoptado términos más amplios como comprensión social (Carpendale y Lewis, 2006) o comprensión sociocognitiva (de Rosnay y Hughes, 2006).

Esta visión del desarrollo sociocognitivo no pretende soslayar la importancia de la comprensión de la falsa creencia, ya que desde luego representa un cambio significativo en la conceptualización infantil de la vida mental. Simplemente, de lo que se trata es de devolver a la noción de TM su sentido evolutivo, haciendo posible explicar sus orígenes y su rol en la emergencia de otras capacidades. A lo largo de este artículo, por lo tanto, asumiremos una visión amplia y gradualista del desarrollo de la TM, refiriéndonos a ella también como comprensión social o comprensión sociocognitiva.

En los últimos años, el interés por explicar las diferencias individuales en el desarrollo de la comprensión social ha motivado una creciente preocupación por las relaciones entre la emergencia de la TM y otros procesos evolutivos. En particular, la numerosa evidencia empírica recogida hasta la fecha señala que son las capacidades lingüísticas las más ampliamente correlacionadas con las habilidades sociocognitivas, particularmente las implicadas en la comprensión de la falsa creencia (véase Milligan, Astington y Dack, 2007, para un meta análisis).

Si nos limitamos al ámbito de las relaciones entre lenguaje y comprensión de la falsa creencia, podemos agrupar parte de la literatura existente en dos grandes líneas de investigación. La primera de ellas plantea que las correlaciones halladas dependen de un tercer factor subyacente, responsable del crecimiento simultáneo de las habilidades lingüísticas y sociocognitivas durante la edad preescolar. Dentro de esta línea, a su vez, podemos diferenciar distintas versiones según el nivel de especificidad del mencionado "factor común". Mientras que algunos autores lo refieren a mecanismos centrales de procesamiento tales como el control inhibitorio (Carlson, Moses y Hix, 1998; Leslie y Polizzi, 1998; Russell, Mauthner, Sharpe y Tidswell, 1991), o la flexibilidad cognitiva (Jacques y Zelazo, 2005; Zelazo y Frye, 1997), otros sugieren que se trata de habilidades más específicas para la manipulación de representaciones mentales, tales como el desdoblamiento representacional (decoupling, Leslie, 1987), la metarrepresentación (Perner, 1991), el proceso de redescripción representacional (Karmiloff-Smith, 1992) o la suspensión semiótica (Rivière y Español, 2003).

Más allá de sus diversos matices, lo que comparten estas teorías es el énfasis en un aspecto previo, de naturaleza cognitiva, que daría cuenta de los estrechos vínculos entre habilidades lingüísticas y comprensión de la falsa creencia.

Sin embargo, un buen número de argumentos provenientes de distintos ámbitos de investigación ha conducido a proponer una segunda hipótesis según la cual existiría una relación de implicación directa entre lenguaje y TM. En primer lugar, más de tres déca- 
das de investigación con primates no humanos permite concluir que las habilidades mentalistas más complejas como la atribución de creencias resultan imposibles en criaturas sin lenguaje (Call y Tomasello, 2008). En segundo lugar, en un sentido más amplio, las primeras evidencias de TM en humanos coinciden con los comienzos de la adquisición del lenguaje. A su vez, el período de mayor desarrollo en TM es al mismo tiempo el período de desarrollo y consolidación de las capacidades lingüísticas, razón por la cual sería esperable, al menos, algún tipo de influencia recíproca. Por último, probablemente los argumentos más sugerentes a favor de una vinculación directa entre lenguaje y TM provengan del estudio del desarrollo atípico. Por una parte, los niños con autismo, que habitualmente fallan en tareas de TM, suelen presentar retrasos lingüísticos o patrones de desempeño atípico (Happé, 1995). Incluso, al examinar más detenidamente las condiciones que hacen posible que algunos niños autistas superen las tareas estándar de TM, la evidencia longitudinal señala que el nivel de habilidad verbal constituye el indicador más poderoso del desempeño en dichas tareas (Tager-Flusberg y Joseph, 2005). Por otra parte, la numerosa evidencia reunida a partir del estudio de los niños sordos demuestra firmemente que aquellos sujetos que presentan dificultades para el razonamiento mentalista presentan también retrasos significativos en su desempeño lingüístico, independientemente de su inteligencia no verbal, de su ajuste sociocognitivo e incluso de su actuación en tareas de función ejecutiva (P. de Villiers, 2005).

En conclusión, los numerosos resultados provenientes tanto de la investigación comparativa como del desarrollo normal y atípico nos llevan a examinar más detenidamente la naturaleza y alcance de esta posible relación de implicación directa entre lenguaje y TM. Asimismo, dentro de esta propuesta podríamos distinguir diferentes versiones según la relación se dirija con más preponderancia de la TM al lenguaje o del lenguaje a la TM. Es esta última formulación causal la que ha recibido mayor apoyo empírico por parte de los investigadores, y al mismo tiempo ha sido objeto de intensos debates teóricos acerca del rol preciso que le corresponde al lenguaje en el desarrollo de la TM (Astington y Baird, 2005a),

En este trabajo, realizaremos una revisión de las principales propuestas teóricas y metodológicas formuladas bajo el supuesto de una vinculación directa entre el desarrollo del lenguaje y el desarrollo de la TM. Comenzaremos revisando los principales antecedentes acerca de los fundamentos sociocognitivos que apoyan la emergencia del primer desarrollo léxico. A continuación, invertiremos la dirección del análisis causal para pasar a considerar las dos principales vías de influencia por las cuales se ha propuesto que el lenguaje propicia la emergencia de la TM. Primeramente, nos detendremos en el papel de los intercambios discursivos en ciertas situaciones sociales privilegiadas, la llamada hipótesis comunicativa, y en segundo lugar pasaremos a considerar las propias habilidades lingüísticas del niño, en particular el rol de ciertas estructuras sintácticas, a través de una revisión de algunos estudios englobados en la denominada hipótesis del determinismo lingüístico. Realizaremos, además, algunas consideraciones metodológicas, e integraremos ciertas sugerencias recientes que podrían abrir futuras líneas de investigación. En tercer lugar, y en consonancia con una perspectiva amplia acerca del desarrollo sociocognitivo, analizaremos brevemente algunas posibles implicaciones del desarrollo de la TM "más allá” de la falsa creencia. Finalmente, en las conclusiones retomaremos el problema de la posible interrelación entre las dos vías de facilitación del lenguaje anteriormente examinadas. Al mismo tiempo, defenderemos la posibilidad de una causalidad recíproca, donde los vínculos entre lenguaje y TM variarán según el momento del desarrollo considerado, y propondremos algunos interrogantes a nuestro juicio abiertos para la investigación futura.

\section{La comprensión social inicial y su influencia sobre las habilidades lingüísticas}

Sobre el final del primer año de vida, numerosas investigaciones han dado cuenta de un cambio cualitativo en la manera en que los niños perciben su mundo social. Indicio 
de ello son algunas conductas frecuentemente observadas, como seguir la dirección de la mirada del otro (Butterworth, 1994), la referencia social (Sorce, Emde, Campos y Klinnert, 1985) y la utilización de gestos de indicación (Carpenter, Nagell y Tomasello, 1998). Esta incipiente habilidad para coordinar la atención con los demás ha sido referida bajo el término ya clásico de atención conjunta.

Numerosos autores desde el ámbito de la TM no tardaron en proponer que el nacimiento de las intenciones comunicativas y sus conductas concomitantes podría suponer el primer indicio de una atribución de estados mentales simples (Bretherton, 1991; Charman et al., 2000; Tomasello y Carpenter, 2007; Wellman, Phillips, Dunphy-Lelii y Lalonde, 2004). Tomasello y colaboradores, por ejemplo, sugieren que los procedimientos de atención conjunta suponen una capacidad cognitiva y motivacional única para la "intencionalidad compartida". Dicha capacidad permite al individuo aprender a través de la interacción cooperativa y compartir estados mentales, tanto para la resolución de problemas como para la comunicación (Tomasello y Carpenter, 2007). Desde esta perspectiva, la aparición de ciertas conductas características como el gesto de indicación, por ejemplo, no obedecería únicamente a razones conductuales, como querer orientar físicamente al otro hacia objetos del campo perceptivo, sino que más bien se trataría de orientarlo "mentalmente", de compartir un campo de experiencias que trasciende la realidad actual (Behne, Carpenter y Tomasello, 2005; Tomasello, Carpenter y Liszkowski, 2007).

Al igual que el gesto de indicación, el aprendizaje léxico tiene lugar en el marco de la interacción entre el adulto, el niño y los eventos del entorno. Por consiguiente, las habilidades lingüísticas iniciales emergen a partir de las mismas habilidades sociocognitivas y motivacionales ya presentes en el acto de indicación. Así, el establecimiento del significado no sería únicamente una cuestión de hacer corresponder referentes y unidades léxicas a partir de una serie de restricciones cognitivas, sino de captar la intención referencial del hablante en una situación determinada.

¿Cómo consiguen los niños capturar la intención referencial del hablante? Tomasello (2001) sugiere que son las habilidades sociocognitivas tempranas las que permiten al niño valerse de ciertas claves socio-pragmáticas que lo orientarán en la búsqueda del significado.

Una de las primeras claves es la que proporciona la dirección de la mirada. Algunos estudios han podido comprobar que alrededor de los dieciocho meses las estrategias de denominación seguidas por la madre se acompañan, por parte del niño, de una subsecuente adaptación al foco atencional del adulto (Baldwin, 1995).

Ahora bien, dado que las situaciones de interacción en las que se produce el aprendizaje de palabras son habitualmente más complejas que la denominación de objetos y acciones en un espacio referencial limitado, la dirección de la mirada no podría ser la única fuente de información. En la última década, Tomasello y sus colaboradores han llevado a cabo una serie de estudios en los que demostraban que la atribución de intencionalidad a las acciones de los otros constituye una fuente importante de información a la hora de identificar el referente para un término desconocido. En una tarea de búsqueda de objetos, el experimentador proponía a niños entre 18 y 24 meses "buscar el toma". Se trataba de averiguar cómo el niño, en ausencia de otras claves facilitadoras como la dirección de la mirada, llegaría a identificar el referente para la palabra nueva. Los resultados indicaron que la mayor parte de las veces los niños comprendían que el referente era el objeto que el experimentador se proponía encontrar y frente al cual mostraba haber cumplido su propósito, independientemente de que el objeto en cuestión fuera el primero en ser encontrado o que la búsqueda concluyera luego de varios intentos fallidos (Tomasello y Barton, 1994). La relación entre actividad referencial e interpretación intencional de la conducta ha podido demostrarse, incluso, en la paradójica situación en la cual, al no poder encontrar el objeto, el adulto mostraba disgusto en lugar de alegría, y el niño no tenía oportunidad de ver el referente luego de haber escuchado su nombre. 
Aún en este caso, los niños comprendían que el "toma" era el objeto que el adulto no había conseguido encontrar (Akhtar y Tomasello, 1996). Finalmente, el rol de la comprensión intencional ha demostrado ser especialmente crucial para el caso del aprendizaje de verbos, ya que se trata de una categoría léxica que se aprende en contextos interactivos por excelencia y nunca de una forma ostensiva (Akhtar y Tomasello, 1996; Tomasello y Barton, 1994).

Además de comprender la intencionalidad de la conducta, las habilidades sociocognitivas surgidas después del primer año permiten al niño construir junto al interlocutor una base de conocimiento común para la comunicación. En relación con ello, Akhtar y Tomasello (1996) también mostraron que alrededor de los dos años los niños son capaces de aprender nuevas palabras valiéndose de la diferenciación entre aquello que es nuevo y aquello que forma parte de las experiencias compartidas con el adulto. El compartir o no un marco de atención conjunta es una clave sociopragmática que ha demostrado influenciar no sólo el desarrollo léxico inicial sino también la capacidad para formular peticiones, una vez las habilidades lingüísticas del niño se vuelven más complejas (ONeill, 1996).

Por consiguiente, podemos concluir que las primeras habilidades sociocognitivas, tal como se expresan en las actividades de atención conjunta, resultan críticas para el aprendizaje léxico inicial. A este respecto, cabe realizar dos puntualizaciones importantes. Por una parte, en las edades que estamos considerando, el crecimiento léxico constituye la manifestación más evidente de las capacidades lingüísticas. Los niños se inician en el lenguaje aprendiendo palabras, y aunque comprenden e intentan producir enunciados completos, sus producciones iniciales se componen de palabras aisladas y de las denominadas holofrases, que vehiculan la intención global de lo que sería una expresión adulta (ver Tomasello, 2006 para una explicación completa). Por este motivo, la influencia de la TM en el lenguaje antes de los dos años de edad solamente puede plasmarse en el vocabulario y, en todo caso, en algunas combinaciones iniciales del mismo. Por otra parte, a pesar de que el léxico únicamente constituye una dimensión del conocimiento lingüístico, desde ciertas propuestas explicativas supone la base para la emergencia de las otras dimensiones (Bates y Goodman, 1997).

Un argumento importante a considerar es el de la relación entre atención conjunta y desarrollo del lenguaje en el autismo. Se ha señalado que los niños autistas, quienes tienen dificultades con la atención conjunta, también muestran dificultades en el aprendizaje del lenguaje (Carpenter y Tomasello, 2000), especialmente si se les compara con niños con otros trastornos, quienes presentan dificultades cognitivas generales, pero no deficiencias en la atención conjunta (Baron-Cohen, Baldwin y Crowson, 1997). Puede aducirse que a pesar de que en los niños con autismo se observa un retraso o ausencia de conductas de atención conjunta, en algunos casos desarrollan un lenguaje acorde con su edad. Sin embargo, consideramos que esta observación no invalida la relación entre la atención conjunta y el desarrollo inicial del lenguaje, puesto que algunos datos apuntan que los niños con autismo que tienen mejores habilidades de atención conjunta son los que luego desarrollan un lenguaje más apropiado a su edad (Charman, 2003; Charman et al, 2003).

A lo largo de este apartado, hemos intentado fundamentar cómo las primeras formas de conocimiento social, ligadas a la comprensión del carácter intencional de las acciones de los otros y a la habilidad para compartir información constituyen uno de los pilares que hacen posible la emergencia del lenguaje. Anteriormente, además, habíamos mencionado que las capacidades para compartir la atención y la intención con otros han sido identificadas como los precursores más tempranos de la TM. Ahora bien, ¿cómo se produce el pasaje desde estas formas originarias de cognición social a aquellas que posibilitan comprender la conducta de los otros en términos de sus representaciones mentales? ¿Qué papel juega el lenguaje en dicho proceso? Una vez los niños comienzan a usar el lenguaje en contextos comunicativos cada vez más complejos, se vuelven también capa- 
ces de incorporar las experiencias sociales y las categorías lingüísticas que posibilitan el acceso al mundo mental. En el siguiente apartado, por tanto, abordaremos el papel que dichas experiencias y categorías puede tener sobre la adquisición de modos más sofisticados de comprensión interpersonal.

\section{El intercambio conversacional como vía de acceso al mundo mental}

En 1991, Judy Dunn y sus colaboradores (Dunn, Brown, Slomkowski, Tesla y Youngblade, 1991) publicaron el primer trabajo en el que se exploraban las posibles relaciones entre el desarrollo de la TM y la calidad de las interacciones en el contexto familiar. Los resultados de este primer estudio sugerían que los niños que en sus hogares estaban expuestos a una mayor cantidad de conversaciones acerca de las causas psicológicas de la conducta eran aquellos que siete meses más tarde mostrarían ser más avanzados en la atribución de creencias, independientemente de sus habilidades lingüísticas y de la cantidad de intercambios conversacionales en la familia. Estos hallazgos motivaron un creciente interés por identificar las condiciones del contexto y las formas de interacción social capaces de precipitar una comprensión más sofisticada de la vida mental.

En los últimos 15 años, el ámbito de la TM ha asistido a la expansión de una línea de investigación centrada en demostrar que ciertas experiencias sociales, como el número de hermanos en la familia (Perner, Ruffman y Leekam, 1994) la presencia de juego de ficción (Astington y Jenkins, 1995); la rememoración conjunta de hechos pasados (Lagattuta y Wellman, 2002) o la narración de historias (Adrian, Clemente y Villanueva, 2007) representan contextos facilitadores para el desarrollo de la comprensión de la mente en el niño. Más allá de la mayor o menor solidez de sus resultados, todos estos estudios asocian las variables estudiadas con la presencia de un cierto estilo de interacción conversacional, el cual a su vez repercutiría sobre las capacidades mentalistas de los niños.

La llamada "bipótesis comunicativa" sostiene que es a través de la participación en interacciones sociales mediadas por el uso del lenguaje como los niños se hacen competentes en la utilización de términos psicológicos para explicar la conducta (de Rosnay y Hughes, 2006; Dunn et al., 1991; Harris, 2005). Este planteamiento pretende rescatar la clásica tesis vygotskiana según la cual las funciones mentales superiores se originan en procesos sociales comunicativos (Vygotski, 1978). Por su propia naturaleza, entonces, esta propuesta supone un verdadero desafío no sólo conceptual sino, sobre todo, metodológico. Se trata de reunir en un único diseño medidas observacionales y experimentales cuyas demandas lingüísticas y cognitivas no son en modo alguno equivalentes. La hipótesis comunicativa se centra en los aspectos más interactivos de la conducta lingüística, de manera tal que cuando se toman medidas del input conversacional, las mismas no evalúan las habilidades lingüísticas individuales sino la construcción conjunta de significados. Al mismo tiempo, las tareas estándar de TM suelen involucrar otras habilidades lingüísticas que es necesario medir y controlar. Esto hace que, en este marco, las relaciones entre lenguaje y TM no siempre sean fácilmente interpretables. Como veremos más adelante, mientras que algunos autores apuntan a una influencia de los aspectos comunicativos sobre los aspectos más representacionales (intraindividuales) del lenguaje, y de allí a las capacidades de TM, otros conjeturan que la estructura del intercambio comunicativo incide directamente sobre la TM, sin necesariamente involucrar aspectos de representación conceptual.

Buena parte de los estudios realizados al abrigo de la hipótesis comunicativa han sido de naturaleza correlacional. En estos términos, resulta imposible determinar si es el tipo de input conversacional el que promueve el desarrollo de la TM, o si, por el contrario, es el nivel de comprensión sociocognitiva del niño el que motiva a los adultos y pares de su entorno a elaborar un tipo de discurso de naturaleza más mentalista. 
En uno de los primeros intentos por aislar el efecto del desempeño en TM sobre la calidad del input, Ruffman, Slade y Crowe (2002) evaluaron a un grupo de niños y sus madres en tres oportunidades a lo largo de un año. En cada momento, los niños fueron evaluados en sus capacidades lingüísticas y su nivel de TM. Al mismo tiempo, se registraron las interacciones discursivas entre madre y niño durante una tarea de descripción de imágenes, lo cual permitía obtener una medida de la densidad de términos de estado mental en el discurso materno, y sus posibles transformaciones a lo largo del tiempo. Los autores encontraron que el número de emisiones mentalistas en el discurso materno en los primeros dos tiempos constituía un predictor importante del posterior desempeño de los niños en las tareas de TM, luego de controlar el efecto de la habilidad lingüística, la cantidad de términos de estado mental o el nivel de TM inicial demostrado por el niño. Más interesante aún, Ruffman et al. determinaron que las relaciones entre input materno y TM no eran recíprocas sino causales, en la medida en que el nivel inicial de TM del niño no contribuyó significativamente a explicar el tipo de discurso materno posterior. Más recientemente, Adrian et al. (2007) extendieron los hallazgos longitudinales de Ruffman et al. a niños mayores, comprobando la eficacia del discurso mentalista materno sobre habilidades más avanzadas de TM.

Otros estudios que han defendido el valor del input conversacional para la emergencia de la TM lo han hecho por la vía de intentar demostrar una contribución exclusiva de la interacción social, más allá de las habilidades lingüísticas del niño. Así, por ejemplo, estudios realizados con niños sordos muestran que aquellos que han adquirido la lengua de signos como lengua materna presentan un desempeño significativamente superior en tareas de TM que los niños sordos de padres oyentes, que habiendo aprendido la lengua de signos de modo tardío, presentaban un acceso muy limitado a las interacciones conversacionales (Peterson y Siegal, 1995). Si bien es cierto que estos primeros resultados no permitían discriminar hasta qué punto las diferencias encontradas podían atribuirse a la pobreza del input o al limitado desarrollo lingüístico esperable en signantes tardíos, existen algunas evidencias posteriores de que la escasa participación en interacciones conversacionales contribuye de manera exclusiva a explicar el retraso observado en tareas de TM (Schick, de Villiers, de Villiers y Hoffmeister, 2007; Woolfe, Want y Siegal, 2002).

En consonancia con una visión amplia y gradualista del desarrollo sociocognitivo, algunos estudios recientes han extendido el alcance de la hipótesis comunicativa al intentar determinar las relaciones entre input materno, lenguaje del niño y habilidades de TM anteriores a la comprensión de la falsa creencia (Ensor y Hughes, 2008; Taumoepeau y Ruffman, 2006; 2008) Los resultados de estos estudios son altamente sugerentes en más de un sentido. Taumoepeau y Ruffman $(2006 ; 2008)$ han encontrado que el lenguaje mentalista materno, específicamente el lenguaje sobre deseos a los 15 meses y sobre conocimientos y creencias a los 24 , posee una influencia exclusiva sobre dos indicadores tempranos del desarrollo sociocognitivo en el niño: la producción de términos mentalistas y el desempeño en una tarea de comprensión de la emoción. Por su parte, Ensor y Hughes (2008) han extendido estos resultados al demostrar que la contingencia semántica de los turnos conversacionales en los que se insertan los términos de estado mental constituye un aspecto crucial para promover la comprensión sociocognitiva en el niño pequeño. De estos estudios interesa destacar dos aspectos importantes. Por un lado, demuestran que el discurso mentalista materno únicamente es eficaz cuando es capaz de ubicarse en la Zona de Desarrollo Próximo del niño, proporcionando los andamiajes adecuados tanto a su nivel de comprensión social como a sus habilidades conversacionales. Este resultado confirma que las medidas conversacionales agregan una dimensión única al análisis de las relaciones entre lenguaje y TM. Por otro lado, el hecho de utilizar medidas más simples y con mínimas demandas verbales, como la tarea de comprensión de la emoción, demuestra que las relación lenguaje-TM trasciende el ámbito 
de la comprensión de la falsa creencia, y no es un simple efecto de la elevada carga lingüística que habitualmente poseen las tareas estándar de TM.

Parece claro, pues, que las interacciones conversacionales son candidatas legítimas a ocupar un rol determinante para la comprensión del mundo mental de las personas. No obstante, creemos necesario precisar qué tipo de input conversacional resulta más propicio para estimular la emergencia de la TM, y cuáles son los contextos que mejor favorecen dichos intercambios.

Ya hemos mencionado el papel de la densidad de términos de estado mental en el discurso materno (Dunn et al., 1991). Sin embargo, autores como Dunn y Brown (1993) entre otros, no tardaron en demostrar que más importante que la cantidad de términos mentalistas es la función que cumplen en el discurso. Dichos autores encontraron que únicamente el discurso materno referido a las causas de los estados emocionales en contextos de humor, juego verbal o consuelo ejercía un efecto positivo sobre la comprensión emocional en el niño siete meses más tarde. Poco después, Denham, Zoller y Couchoud (1994) confirmaron el valor predictivo de las "explicaciones de la emoción” por contraposición a las simples referencias a emociones. Más recientemente, Slaughter, Peterson y Mackintosh (2007) informaron de hallazgos similares respecto de la función explicativa de los términos cognitivos por parte de la madre y su efecto sobre la comprensión de estados cognitivos en el niño. En una dirección similar, Adrian et al. (2007) hallaron que las funciones pragmáticas que realizan las emisiones mentalistas maternas en una situación de narración de historias son factores más sensibles que el número total de términos cognitivos a la hora de predecir el desempeño de los niños en tareas de falsa creencia. Por consiguiente, estos resultados sugieren que el nivel de elaboración del discurso mentalista materno constituye un factor más relevante que la sola frecuencia de una serie ítems léxicos para el desarrollo de la comprensión de la mente en el niño.

El problema de la calidad frente a la cantidad también se ha hecho presente a la hora de identificar el tipo de experiencias más propicias para favorecer la emergencia de conversaciones con contenido mentalista. Hace ya más de una década, Perner et al. (1994) encontraron que aquellos niños con dos hermanos tenían casi el doble de probabilidad de responder correctamente en una tarea de falsa creencia, que aquellos que no tenían hermanos. Poco después, Jenkins y Astington (1996) fueron un poco más allá al proponer que el tamaño de la familia podría tener un efecto compensador sobre el desempeño en TM en aquellos niños con menores capacidades lingüísticas.

Ahora bien, ¿qué elemento del hecho de tener hermanos podría facilitar el desarrollo social? En principio, todos los resultados parecen concordar en que no es la cantidad de hermanos lo relevante sino el tipo de interacciones que el niño tiene mayor probabilidad de experimentar cuando hay hermanos presentes dentro del contexto familiar. Ya a finales de los años 80, Dunn (1988) observaba que los niños con hermanos mayores asisten con mayor asiduidad a intercambios con contenido mentalista entre éstos y sus padres. Otra de las experiencias sociales ligadas a la presencia de hermanos es una mayor frecuencia de situaciones de juego cooperativo. Numerosos estudios han señalado que cuando se trata de juego de ficción, la discusión acerca de los pensamientos y sentimientos de los personajes imaginarios son frecuentes. La presencia de este tipo de interacciones y de ciertos elementos del discurso que las caracterizan (como la asignación de roles, la realización de planes conjuntos o la fantasía compartida) se ha relacionado significativamente con el desempeño de los niños en tareas de TM (Astington y Jenkins, 1995). En contraste, otras medidas relativas a la cantidad total de juego de ficción, como la diversidad de temas o el juego simbólico solitario no correlacionan en la misma medida con la actuación en tareas de TM.

Además de la presencia de hermanos, hay aún otras experiencias sociales dentro del contexto familiar que podrían tener una relación privilegiada con el discurso mentalista. Estudios recientes como el de Lagattuta y Wellman (2002) señalan que las conversacio- 
nes entre padres e hijos acerca de episodios biográficos del pasado tienden a generar un discurso abundante en referencias mentalistas con función explicativa, estimulando a los niños a razonar de modo precoz sobre la causalidad psicológica de la conducta humana. Más allá de los intercambios conversacionales, en los últimos años, autores como Elizabeth Meins y colaboradores, entre otros, han prestado especial atención a una propiedad del discurso materno que han dado en llamar mind-mindedness (término que podría expresarse como "mentalismo materno") para referirse a una tendencia por parte de la madre a describir a sus hijos en términos más mentalistas que físicos, y también a tratarlos como individuos con pensamientos y sentimientos autónomos. En el contexto de un extenso estudio longitudinal, Meins, Fernyough, Russell y Clark-Carter (1998) solicitaron a madres que describieran a sus hijos de tres años de edad. Dichas descripciones fueron categorizadas en términos de atributos mentales, conductuales o físicos. Los autores encontraron que el uso de atribuciones de estado mental por parte de la madre podía predecir la habilidad del niño para comprender falsas creencias a los cuatro años de edad, y la capacidad para atribuir emociones basadas en creencias un año mas tarde. Estos primeros hallazgos fueron confirmados posteriormente en otros contextos y mediante medidas similares de mentalismo materno, las cuales han mostrado ser más potentes que otros indicadores no verbales de la sensibilidad materna como por ejemplo la seguridad del apego (Meins et al., 2002).

La evidencia comentada hasta aquí nos sugiere, en primer lugar, que existen una serie de características del discurso dirigido al niño capaces de promover mejoras en su comprensión de la mente. En segundo lugar, que ciertas experiencias sociales tempranas resultan particularmente propicias para incrementar dichos usos discursivos. Por último, la mayor parte de los estudios destaca el input conversacional de la madre como uno de los predictores más importantes del desempeño de los niños en tareas que evalúan tanto la comprensión de creencias como de emociones a ellas asociadas.

El problema es determinar exactamente cuál es el mecanismo que hace posible que el intercambio conversacional propicie la adquisición de la TM. Se trata de una cuestión de difícil resolución empírica y que ha suscitado, por ende, un intenso debate teórico entre los investigadores del área.

Por una parte, algunos autores han colocado el acento en las funciones pragmáticas de la conversación. Desde este punto de vista, la participación en la conversación permite al niño tomar conciencia de la existencia de diferentes perspectivas (Dunn et al., 1991; Harris, 2005). Harris (2005) propone que más allá de su contenido referencial o de su complejidad formal, es la estructura del intercambio conversacional y las funciones pragmáticas que realizan los participantes en él las que resultan eficaces para el desarrollo de la TM. En particular, son los intentos de la madre por introducir puntos de vista divergentes en la conversación, los que constituyen la fuente subyacente de variación en comprensión de la mente en el niño pequeño.

Un estudio de entrenamiento reciente (Lohmann y Tomasello, 2003) resulta particularmente revelador para esta perspectiva. En dicho estudio, los autores pretendían poner a prueba la influencia conjunta o específica de tres variables sobre la comprensión de la falsa creencia: la experiencia con objetos o situaciones engañosas, la confrontación de perspectivas en la interacción discursiva, y la comprensión de complementos oracionales con verbos mentales y de comunicación. Si bien los resultados de este estudio poseen múltiples implicaciones que retomaremos más adelante, lo que nos interesa destacar aquí es que el hecho de exponer a los niños a una situación de interacción en la que se enfatizaban distintos puntos de vista sobre un mismo objeto fue suficiente para mejorar su rendimiento en tareas de TM, aún en ausencia de términos de estado mental. Incluso, dicha mejora se produjo a pesar de un muy pequeño incremento en la comprensión de complementos oracionales, lo cual permitiría descartar que la eficacia del entrenamiento discursivo radique en cambios en otras habilidades lingüísticas subyacentes. 
Otros autores, sin embargo, han enfatizado los aspectos más semánticos implicados en el input conversacional. Desde esta perspectiva, si la conversación abundante en contenido mentalista es la que ha tenido un efecto, es porque ella posibilita la abstracción de conceptos de estado mental a partir del uso de términos específicos para codificarlos (Bartsch y Wellman, 1995; Olson, 1988). Así, según Olson (1988), la adquisición de términos que se refieren a estados mentales mediatiza el desarrollo de la comprensión de la mente, ya que posibilita extraer analogías entre la propia experiencia y la de los demás. El lenguaje, así, crea categorías que permiten distinguir entidades con un alto grado de opacidad referencial, como por ejemplo las creencias y deseos. De este modo, es en la conversación que los niños y niñas adquieren el lenguaje mental específico que les permite entender los estados mentales y afectivos que regulan la conducta de las personas.

A lo largo de este apartado hemos aportado diversas evidencias que nos permiten concluir que el input discursivo al que el niño está expuesto juega un papel relevante en la emergencia de la TM. Resulta menos claro, sin embargo, cuál es la razón por la cual dicho input produce sus efectos. Probablemente las perspectivas mencionadas no resulten del todo contradictorias. Por una parte, debe señalarse que los resultados del estudio de Lohmann y Tomasello (2003) indican que tanto el intercambio de perspectivas en el discurso como el dominio de las propiedades semántico-sintácticas de los términos de estado mental parecen realizar contribuciones independientes e igualmente importantes al desarrollo de la TM. Por otra parte, la observación naturalista muestra que la frecuencia de verbos mentalistas se relaciona con el uso de cláusulas de complemento y con la tendencia a armar un discurso sobre perspectivas en conflicto. Se trataría de diferentes aspectos del input materno que covarían, y por lo tanto resulta difícil y hasta riesgoso intentar aislarlos en contextos experimentales. De hecho, no resulta casual que en el estudio de entrenamiento anteriormente comentado, la condición más eficaz para producir avances en las capacidades mentalistas de los niños fuera aquella que integraba los tres aspectos mencionados.

No obstante, la historia de la relación entre lenguaje y TM no estaría completa sin definir el rol que en ella juegan las propias habilidades lingüísticas del niño. Algunos autores sostienen, como veremos en el siguiente apartado, que no es el input conversacional lo que propicia la adquisición de la TM, sino que es el desarrollo de las habilidades lingüísticas, particularmente en su dimensión gramatical, las que permiten al niño la participación en la conversación y la emergencia de la TM (Astington y Jenkins, 1999; de Villiers y Pyers, 2002). Dicha opción es la que analizaremos a continuación.

\section{Desarrollo sintáctico y razonamiento mentalista}

Como hemos revisado en el apartado anterior, se han propuesto los niveles semántico y pragmático como aquellos que conducen el desarrollo en TM. No obstante, a partir de un influyente trabajo de Astington y Jenkins (1999) las habilidades sintácticas recibieron creciente consideración al encontrarse particularmente asociadas a la comprensión de la falsa creencia (en adelante, CFC).

No está claro por qué motivo la sintaxis podría ejercer un papel relevante en la CFC. A un nivel muy general, se considera que las habilidades sintácticas posibilitan el razonamiento sobre creencias falsas al facilitar la representación de estados diferentes a la realidad (Plaut y Karmiloff-Smith, 1993). Sin embargo, en la última década, Jill de Villiers y colaboradores han propuesto una hipótesis mucho más especifica (de Villiers, 2005; de Villiers y de Villiers 2000; de Villiers y Pyers, 2002). Dichos autores sostienen que el factor clave para el razonamiento acerca de creencias falsas reside en el dominio infantil de la sintaxis de los complementos oracionales. Los complementos oracionales son construcciones complejas que consisten en una oración principal con otra insertada 
en ella, y que funciona como objeto gramatical del verbo en la oración principal. Esta tipología de oraciones se ilustra seguidamente:

(1) Ada dice que la televisión es de color naranja

(2) Naira pensaba que María le había roto el juguete

Solamente este tipo de complementos dependientes, presentes en oraciones con verbos principales como pensar o decir, tienen la propiedad de que pueden ser falsos, pudiendo ser verdadera la oración que los contiene. La afirmación (1) puede ser cierta en su conjunto y, sin embargo, no ser cierto el contenido de su complemento oracional. De manera similar, en el ejemplo (2), si la cláusula completiva "que María le babía roto el juguete" codifica el contenido de la mente de Naira, la verdad de esta proposición no puede ser valorada con respecto al mundo real, sino en relación con el mundo mental de Naira. Esta propiedad ha conducido a algunos autores a asumir que la complementación oracional proporciona un marco de representación para la CFC.

Lo que es relevante para el desarrollo de la TM es que ante estas oraciones, los niños menores de cuatro años no podrían considerar que la proposición que complementa a la principal sea falsa, a la vez que es verdadera la oración en su conjunto. Alrededor de los 4 años, en cambio, adquirirían esta capacidad. Así pues, los niños que comprenden este tipo de estructuras disponen, por una parte, de un conocimiento sintáctico específico, ya que saben que algunos verbos toman complementos oracionales, pero también disponen de conocimiento sociocognitivo, puesto que saben que otra persona puede tener una creencia que es falsa. Y además, saben que hay una conexión entre esta forma sintáctica y la expresión de creencias potencialmente falsas.

Hay que destacar dos aspectos importantes de la hipótesis propuesta por de Villiers y colegas. En primer lugar, la misma no se aplica a todos los complementos oracionales, sino solamente a los que se encuentran incrustados en verbos mentales y de comunicación que toman complementos sobre sucesos potencialmente reales, también llamados complementos realis. A diferencia de los complementos realis, que pueden ser verdaderos o falsos, los complementos irrealis, característicos de otros verbos mentalistas como prometer, querer o desear, tratan sobre sucesos futuros o hipotéticos, y a los que no puede atribuírsele un valor de verdad determinado. En segundo lugar, se propone que los niños primero dominan los complementos oracionales incrustados en verbos de comunicación, donde puede haber evidencia explícita de que el complemento es falso y luego, por analogía, comprenden los complementos oracionales incrustados bajo verbos mentales (de Villiers, 2005).

Dentro de esta propuesta explicativa, un estudio clave es el de de Villiers y Pyers (2002), realizado con niños de 3 a 5 años a los que se les aplicó una serie de medidas lingüísticas y otras medidas de creencias falsas en cuatro momentos temporales diferentes. Estos autores hallaron que el buen desempeño en estas últimas tareas no estaba relacionado con la habilidad lingüística general medida, por ejemplo, mediante LME u otras medidas sintácticas, sino que se basaba exclusivamente en el dominio de las estructuras que se utilizan para expresar complementos de verbos de comunicación o de pensamiento.

A pesar de algunos resultados inconsistentes, en la actualidad buena parte del debate en relación con el papel que juega el lenguaje en el desarrollo de la TM se encuentra centrado en aportar evidencia a favor o en contra de si el dominio infantil de los complementos oracionales es crucial en el desarrollo de la CFC.

Dentro de las líneas de investigación que han aportado mayor cantidad de evidencia empírica a la hipótesis de la complementación oracional encontramos la realizada con datos obtenidos a partir de la ejecución en tareas de CFC por parte de niños sordos. Gale, de Villiers, de Villiers y Pyers (1996) hallaron que los niños sordos educados en escuelas exclusivamente orales, quienes por tanto ven retrasado su desarrollo lingüístico, tienden a pasar las tareas de CFC unos tres años más tarde que los niños oyentes. En este estudio, al analizar las diferencias individuales en el desarrollo de la TM, se encon- 
tró que el dominio de las estructuras de complementación oracional era el mejor predictor de la ejecución en CFC, incluso tras controlar la habilidad lingüística general. Además, este tipo de habilidades sintácticas ha demostrado ser eficaz aún cuando se elimina el contenido verbal de las tareas de TM. En una dirección similar, de Villiers y de Villiers (2000) presentan resultados de niños sordos que, a pesar de presentar un CI normal y una inteligencia social activa, se encontraban significativamente retrasados tanto en tareas verbales como no verbales de TM. Nuevamente, el desempeño en ambos tipos de tareas podía predecirse mejor por la producción de complementos oracionales con verbos de comunicación o de estado mental, que por la habilidad lingüística general. Recientemente, Schick et al. (2007) han hallado resultados similares al comparar signantes nativos, tardíos y niños sordos "oralizados", aunque cabe puntualizar que también encontraron un efecto específico del tamaño del vocabulario general, que los autores interpretan en la vía de la influencia del input conversacional. Más allá de su apoyo a la hipótesis de la complementación oracional, estos resultados avalan la idea ya sugerida en el metanálisis de Milligan y colaboradores (Milligan et al., 2007) según la cual la relación entre ciertas habilidades lingüísticas y la CFC no resulta de las altas demandas verbales de las tareas en sí, como la complejidad de las preguntas formuladas o la necesidad de seguir una estructura narrativa compleja.

De manera parecida a la investigación con niños sordos, diferentes estudios muestran la relación entre el lenguaje, particularmente la sintaxis, y la TM en niños autistas (Fisher, 2002; Tager-Flusberg y Sullivan, 1994). Según Tager-Flusberg y Joseph (2005), la adquisición de los complementos oracionales con verbos de comunicación es la clave para comprender por qué algunos niños con autismo son capaces de pasar las tareas de falsa creencia. A partir de sus propios datos, estos autores defienden que estos niños se apoyan especialmente en el lenguaje, y en particular en los complementos oracionales, para lograr su capacidad metarepresentacional.

A pesar de las evidencias que hemos comentado, existen algunos estudios que han puesto en cuestión el rol de los complementos oracionales para la CFC. En estos estudios se ha observado que la semántica, pero no la sintaxis, predice la puntuación en tareas de TM (Ruffman, Slade, Rowlandson, Rumsey y Garnham, 2003) o que algunas medidas lingüísticas más amplias funcionan como mejores predictores de la CFC (Slade y Ruffman, 2005). En concreto, Slade y Ruffman (2005) examinaron las asociaciones longitudinales entre tres tareas de CFC, una tarea de memoria y cuatro tareas de lenguaje. Sus resultados indicaron no sólo una relación bidireccional entre el lenguaje y TM, sino que, a diferencia de otros estudios, ninguna medida de sintaxis o semántica predijo más que otra la ejecución posterior en TM. Otros trabajos, como el de Cheung et al. (2004) también han puesto en cuestión la generalización de los resultados sobre complementación oracional. Al aplicar una medida diferente de la memoria para complementos, estos autores no han encontrado una contribución única de este tipo de estructuras sobre la CFC. Por último, otros autores han apelado a las variaciones translingüísticas en las formas de complementación para cuestionar que el dominio de la complementación oracional sea el único rasgo del lenguaje implicado de manera importante en el desarrollo de la TM (Perner, Sprung, Zauner y Haider, 2003; Tardif y Wellman, 2000) (para una discusión de estos resultados, ver de Villiers, 2005).

Como respuesta a los hallazgos de de Villiers y sus colaboradores, algunos de estos autores plantean que las relaciones encontradas por aquéllos no son sorprendentes, ya que habría un solapamiento entre los requerimientos para las tareas de CFC y las de memoria para complementos (Cheung et al., 2004; Ruffman et al., 2003). El test de memoria para los complementos solicita a los niños que recuerden complementos oracionales falsos incrustados bajo un verbo mental principal (pensar, creer) o comunicativo (decir), como se ilustra en la oración (3).

(3) Él pensó (o dijo) que dibujaría una casa, pero en realidad era una flor. 
De hecho, Ruffman et al. (2003) consideran que los niños no pueden responder por el contenido de la creencia o la enunciación ("¿que pensó (o dijo) que dibujaría?") a menos que comprendan la falsa creencia. Si bien de Villiers (2005) ha discutido este punto, particularmente para los ítems que usan verbos de comunicación, numerosas evidencias sugieren que los niños necesitarían comprender la falsa creencia incluso para responder correctamente a una cuestión sobre lo que alguien dijo, en los casos en que el niño sabe que la persona sostiene una afirmación falsa. Como ha quedado confirmado en un metaanálisis bastante reciente (Wellman, Cross y Watson, 2001), no parecen existir diferencias significativas entre las preguntas de FC usando verbos de comunicación o mentales, diferencias que tampoco se hallaron en el estudio de entrenamiento de Lohmann y Tomasello (2003).

Algunas de las divergencias que muestran los diferentes estudios acerca del rol de la sintaxis en la CFC pueden deberse a las diversas maneras de medir la sintaxis o a cómo se mide la complementación oracional. También al hecho de medir la sintaxis de manera general o centrándose en complementos oracionales de determinado tipo. Hay que tener en cuenta, por ejemplo, que las medidas sintácticas generales pueden correlacionar en gran medida con las medidas sintácticas particulares (como las de los complementos). Así, en algunos estudios se utilizan oraciones completivas pero no con verbos de comunicación o cognición (Cheung et al., 2004); en otros estudios se toman medidas de sintaxis general (de Villiers y Pyers, 2002) y otros estudios se centran en la producción de oraciones completivas (Tager-Flusberg y Joseph, 2005), de manera que los resultados no son demasiado comparables. Por ello es importante tener en cuenta un reciente meta-análisis acerca del papel del lenguaje en el desarrollo de la TM (Milligan et al., 2007). En este meta-análisis se ofrecen resultados que, si bien son favorables a la hipótesis de la complementación, no permiten descartar la influencia de otras habilidades lingüísticas más generales. En concreto, el análisis conjunto de los datos provenientes de distintas investigaciones demostró que la relación entre CFC y el desempeño en diversas tareas de memoria de complementos fue la más elevada, aunque su magnitud no fue significativamente diferente de la encontrada para otras medidas lingüísticas. Para matizar esta aportación, hay que destacar que entre los estudios incluidos en dicho meta-análisis, aquellos que utilizaron una medida de complementación oracional fueron solamente cuatro.

Precisamente, entre las investigaciones incluidas en el meta-análisis de Milligan et al. (2007), destacan dos estudios de entrenamiento que han intentado contrastar la hipótesis de la complementación oracional con otras explicaciones alternativas (Hale y Tager-Flusberg, 2003; Lohmann y Tomasello, 2003). En el primero de ellos, Hale y Tager-Flusberg (2003), encontraron que el entrenamiento en complementos oracionales de tipo completivo comportaba una mejora en la realización de diversas tareas de TM. Además, esta influencia fue muy específica, ya que no ocurría lo mismo en sujetos entrenados en otro tipo de oraciones incrustadas como son las relativas (oraciones que complementan al nombre). No obstante, este estudio aporta aún otros resultados interesantes que vale le pena destacar. Por una parte, las autoras encontraron que el entrenamiento en CFC también mejoraba la realización en CFC, aunque no implicara entrenamiento lingüístico específico. Por otra parte, debido a que durante el entrenamiento en completivas sólo se utilizaron verbos de comunicación, el avance en TM observado en el pos test no podía atribuirse a un efecto de aprendizaje del significado de determinados verbos de contenido mental.

Por su parte, y como ya fuera mencionado en el apartado anterior, Lohmann y Tomasello (2003) encontraron que la condición de entrenamiento más eficaz para promover avances en TM era aquella en la cual se enfatizaban distintas perspectivas sobre un mismo objeto mediante la utilización de verbos de estado mental con complementos oracionales. Sin embargo, hubo además otras dos condiciones que produjeron efectos de manera independiente. Por una parte, el grupo entrenado en completivas mejoró su rea- 
lización en tareas de CFC posteriores, aún cuando dichas cláusulas no enfatizaban el carácter engañoso de los objetos. Por otra parte, también mejoró su realización posterior el grupo entrenado en la confrontación de perspectivas durante la conversación. Así pues, estos datos permiten sostener que si bien el entrenamiento en complementación sintáctica parece ser suficiente para provocar avances en la TM, no es posible concluir que sea el único factor influyente.

En síntesis, todo parece indicar que el debate acerca del papel de la sintaxis en la CFC está lejos de haberse cerrado. Aunque los planteamientos iniciales de de Villiers enfatizaban las propiedades sintácticas de la complementación como prerrequisito para razonar sobre falsas creencias (de Villiers y de Villiers, 2000), más recientemente la propia autora ha debido introducir algunas restricciones relativas a la semántica de los verbos de creencia y comunicación (de Villiers, 2005).

Probablemente, la propiedad semántica del tipo que hemos descrito para las oraciones completivas sea crucial para proporcionar a los sujetos los medios para representar explícitamente la dependencia de una afirmación falsa en la construcción completiva. Sin embargo, aún admitiendo la importancia de la complementación oracional, otros autores consideran que no es la única propiedad del lenguaje que permite el acceso a la CFC. Otros candidatos sobre los que merece la pena profundizar en la investigación futura son la participación discursiva a la que ya hemos hecho referencia (Lohmann y Tomasello, 2003) o la habilidad para considerar múltiples nombres para los objetos (Perner et al., 2003).

\section{Después de la falsa creencia: el impacto de la TM sobre fenómenos lingüísticos y comunicativos complejos}

La mayoría de las investigaciones sobre la relación entre TM y lenguaje se limitan a los años preescolares. Sin embargo, con la CFC lo que el niño adquiere es el reconocimiento de que puede mantenerse una falsa creencia sobre las cosas del mundo real. Pero para poder comprender el mundo de las relaciones interpersonales en toda su complejidad, los niños mayores necesitarán dar un paso más para llegar a concebir que los otros también pueden tener "creencias sobre las creencias" de los demás (Filippova y Astington, 2008).

¿Qué sucede, pues, más allá de la CFC? ¿Cómo podemos pensar las relaciones entre TM y lenguaje más allá de los años preescolares?

Uno de los aspectos más estudiados a propósito de las conquistas "post-falsa creencia" se vincula a la capacidad de los sujetos para hacer uso de una de las propiedades más características de los enunciados de estado mental: su recursividad. Perner (1988), por ejemplo, ha propuesto que el principal avance después de los años preescolares se presenta en un aumento del número de recursiones que se pueden operar con respecto a los estados intencionales. A diferencia de las atribuciones de primer orden ("A cree que la canica está en la canasta"), las llamadas creencias de segundo orden ("A piensa que B piensa que la canica está en la canasta") suelen comprenderse con un desfase de dos años aproximadamente (Perner, 1988), lo cual no deja dudas respecto a su complejidad Algo similar sucede con las intenciones acerca de creencias (“A quiere que B crea que...").

Indudablemente, esta habilidad para manejar un mayor número de recursiones juega un rol central en el refinamiento de las capacidades mentalistas que demuestran los sujetos durante sus años escolares. En particular, abre el camino a una variedad más compleja de interacciones comunicativas, ya que posibilita el sostenimiento de actitudes hacia las actitudes de los demás. Al mismo tiempo, es evidente que el desarrollo de las capacidades lingüísticas en todas sus dimensiones contribuye a enriquecer este proceso, aunque probablemente sean las habilidades pragmáticas las más estrechamente relacionadas con los mencionados cambios en la comprensión de la vida mental. 
La adquisición del razonamiento de segundo orden se ha relacionado con la capacidad para comprender actos de habla indirectos. En particular, el uso de la metáfora, la ironía y la mentira han sido vías privilegiadas para el estudio de las habilidades pragmáticas y sus posibles vínculos con el desarrollo de la TM más allá de la edad preescolar.

Happé (1993), por ejemplo, estudió la relación entre los diferentes niveles de complejidad implicados en la comprensión de la metáfora y la ironía y la resolución de tareas de falsa creencia de primer y segundo orden. Sus resultados mostraron que mientras que la falsa creencia de primer orden parece ser suficiente para reconocer el significado de la metáfora, la ironía requiere la comprensión de la falsa creencia de segundo orden. Estos datos se han interpretado comparando el carácter descriptivo de la metáfora frente al carácter interpretativo de la ironía, en el sentido de que el oyente necesita considerar los estados mentales del hablante. En una dirección similar, Winner y Leekam (1991) relacionaron la capacidad de los niños para distinguir entre un comentario irónico y una mentira "piadosa”, y el nivel de desempeño en diversas tareas de TM. Los resultados mostraron que para que los niños distinguieran la actitud que hay detrás de la ironía de aquella que hay detrás de una mentira "piadosa”, es necesario que diferencien cuál es la intención del hablante respecto de los estados mentales del destinatario (qué quiere $\mathrm{X}$ que crea $\mathrm{Y}$ ), y por lo tanto, que comprendan situaciones de falsa creencia de nivel superior.

Los datos presentados no parecen dejar dudas acerca de la estrecha interdependencia entre TM y capacidades pragmáticas complejas. Sin embargo, pocos han sido los estudios que han intentado delimitar la contribución específica de las habilidades lingüísticas y el razonamiento mentalista sobre las mencionadas capacidades. Recientemente, Filippova y Astington (2008) han estudiado la evolución del razonamiento social avanzado a partir de la comprensión de la ironía en niños de 5 a 9 años de edad. De manera interesante, sus resultados muestran que tanto la competencia lingüística como las habilidades avanzadas de TM realizan contribuciones únicas a la habilidad para interpretar enunciados irónicos, independientemente de la edad, la memoria y las claves prosódicas que los acompañan. No obstante, lo que no queda claro a partir de estos datos es cuál sería específicamente la contribución del lenguaje en esta tarea, sobre todo si tenemos en cuenta que sólo se ha utilizado una medida de vocabulario receptivo, demasiado general e imprecisa como para avanzar conjeturas más detalladas. En cualquier caso, lo interesante de este estudio, según las propias autoras, es que la ironía y el habla no literal se revelan como un importante medio para estudiar las complejas relaciones entre lenguaje, TM y razonamiento social, lo cual abre un camino prometedor hacia futuras investigaciones que nos permitan avanzar por terreno firme más allá de la edad preescolar.

\section{Conclusiones}

En este trabajo hemos revisado las principales líneas de investigación acerca de las relaciones entre lenguaje y TM. Por una parte, según sugiere la hipótesis comunicativa, es a través de la participación en interacciones sociales mediadas por el uso del lenguaje cómo los niños se hacen competentes en la utilización de términos psicológicos para explicar la conducta. Por otra parte, otros autores han privilegiado las propias habilidades lingüísticas del niño (en particular, la sintaxis de las cláusulas de complemento) como el factor que permite explicar la CFC.

Ahora bien, ¿hasta qué punto estas perspectivas son excluyentes? Para responder a esta pregunta, debemos considerar una serie de cuestiones. En primer lugar, la hipótesis comunicativa asume una perspectiva amplia acerca del desarrollo de la cognición social, mientras que la hipótesis más específicamente lingüística, sin negar la existencia de otras habilidades sociocognitivas, se centra en el desarrollo de un aspecto específico, la CFC. Si, como mencionábamos en la introducción, entendemos las habilidades socio- 
cognitivas en términos de un desarrollo amplio y gradual, podemos considerar que la CFC es un hito particular dentro de este desarrollo, y por lo tanto ambas hipótesis no son incompatibles En segundo lugar, y siguiendo una propuesta de Astington y Baird (2005b), para comprender la influencia del lenguaje sobre la TM es necesario abordar el desarrollo lingüístico desde su doble vertiente, comunicativa y representacional. Esta misma distinción funcional puede utilizarse para diferenciar entre ambas hipótesis causales: mientras que la primera de ellas enfatiza la función comunicativa e interpersonal del lenguaje, la segunda hace hincapié en la función del lenguaje en tanto que instrumento de representación intraindividual. Justamente, si consideramos que estas dos funciones no son contrapuestas sino complementarias, podemos concluir que las dos hipótesis que estamos considerando son dos versiones igualmente complementarias acerca del papel facilitador del lenguaje para la emergencia de la TM.

Resulta claro que tanto el uso de cláusulas de complemento como el input conversacional tienen un papel importante en la adquisición de la TM. Sin embargo, ¿Cómo situar estas influencias en el marco más amplio del desarrollo ontogenético? Todo parece apuntar hacia un esquema de causalidad recíproca, donde los vínculos entre lenguaje y TM variarán según el momento del desarrollo considerado. En un primer nivel del desarrollo sociocognitivo, las nuevas capacidades para compartir la atención con otros e interpretar su conducta en términos intencionales otorgan a los niños una habilidad crucial para la emergencia del primer lenguaje. En esta línea consideramos que, en un sentido amplio, las primeras habilidades de cognición social (precursoras de la posterior TM) constituyen uno de los factores causales del desarrollo del lenguaje inicial, especialmente en sus aspectos léxico-semánticos y pragmáticos. A su vez, el uso del lenguaje en contextos comunicativos cada vez más complejos, junto a la ampliación de sus posibilidades de representación, crea unas condiciones privilegiadas para enriquecer el desarrollo sociocognitivo: lleva a comprender a los otros como agentes mentales, cuya conducta es gobernada por deseos y creencias. Como sugieren Astington y Baird (2005b), la habilidad pragmática permite que los niños participen en intercambios comunicativos, en donde simultáneamente adquieren conciencia de la existencia de diferentes puntos de vista, y del significado y uso de diferentes términos de estado mental. Por otra parte, el creciente dominio de la sintaxis posibilita a los niños crear un marco representacional adecuado para comprender falsas creencias. Posteriormente, los pocos datos con los que contamos en la actualidad sugieren la existencia de una implicación mutua en lugar de un vínculo de causalidad claro entre el crecimiento de las habilidades lingüísticas, en especial en el ámbito pragmático, y el desarrollo de modos cada vez más sofisticados de razonar acerca del mundo interpersonal y mental.

¿Cuáles creemos que podrían ser las vías abiertas para la investigación futura en este ámbito? De acuerdo a algunos datos recientes, podríamos situar dos vertientes principales en las que se están centrando los estudios sobre la influencia del lenguaje sobre el desarrollo sociocognitivo. Por una parte, retomando nociones ya clásicas en la perspectiva sociocultural, como Zona de Desarrollo Próximo o andamiaje, algunos autores intentan clarificar los modos de influencia del input discursivo sobre las habilidades sociocognitivas previas a la CFC (Ensor y Hughes, 2008; Taumoepeau y Ruffman, 2008). En una dirección complementaria, creemos interesante profundizar en cómo las propias habilidades lingüísticas del niño en las primeras fases de su desarrollo gramatical podrían influir sobre dichas habilidades básicas de cognición social, como la comprensión de estados emocionales o del deseo como causa de la conducta. En el otro extremo del desarrollo, otros datos igualmente recientes apuntan a desentrañar las relaciones entre las habilidades lingüísticas, el razonamiento social y la llamada TM avanzada (Filippova y Astington, 2008). Este segundo ámbito de investigación ha sido el menos explorado y resulta particularmente prometedor de cara a la investigación futura. Por una parte, comprender cómo el lenguaje y la TM hacen posible el desarrollo de habilidades sociales complejas nos permitiría explicar mejor las grandes diferencias individuales en este 
ámbito. Por otra parte, aún es necesario delimitar con mayor precisión cuáles son los aspectos del lenguaje implicados en estas nuevas habilidades pragmáticas, y cómo se relacionan con las capacidades mentalistas "más allá de la falsa creencia”.

\section{Referencias}

Adrian, J. E., Clemente, R. \& Villanueva, L. (2007). Mothers' use of cognitive state verbs in picture-book reading and the development of children's understanding of mind: a longitudinal study. Child Development, 78 (4), 1052-1067.

AKHTAR, N. \& TOMASELlo, M. (1996). Twenty four-month-old children learn words for absent objects and actions. British Journal of Developmental Psychology, 14, 79-93.

AstingTON, J. \& JENKINS, J. (1995). Theory of mind development and social understanding. Cognition and Emotion, 9, 151165.

Astington, J. \& JenKins, J. (1999). A longitudinal study of the relation between language and theory-of-mind development. Developmental Psychology, 35, 1311-1320.

Astington, J. W. \& BAIRD, J. A. (Eds.) (2005a). Why language matters for theory of mind. Nueva York: Oxford University Press.

Astington, J. W. \& Baird, J. A. (2005b). Introduction. En J. W. Astington \& J. A. Baird (Eds.), Why language matters for theory of mind (pp. 3-25). Nueva York: Oxford University Press.

BALDWIN, D. (1995). Understanding the link between joint attention and language. En C. Moore \& P. Dunham (Eds.), Joint attention: its origins and role in development (pp. 131-158). Hillsdale, NJ: LEA.

BARon-Cohen, S., BALDwin, D. \& Crowson, M. (1997). Do children with autism use the speaker's direction of gaze strategy to crack the code of language? Child Development, 68, 48-57.

BartSCH, K. \& WelLman, H. M. (1995). Children talk about the mind. Nueva York: Oxford University Press,

BATES, E. \& GOODMAN, J. (1997). On the inseparability of grammar and the lexicon: Evidence from acquisition, aphasia and real-time processing. Language and Cognitive Processes, 12 (5/6), 507-586.

Behne, T., CARPENTER, M. \& TOMASELlO, M. (2005). One-year olds comprehend the communicative intentions behind gestures in a hiding game. Developmental Science, 8, 492-499.

BRETHERTON, I. (1991). Intentional communication and the development of an understanding of mind. En D. Frye \& Ch. Moore (Eds.), Children's theories of mind: mental states and social understanding (pp. 49-75). Hillsdale, NJ: L.E.A.

Butterworth, G. (1994). Theory of Mind and the facts of embodiment. En C. Lewis \& P. Mitchell (Eds.), Children's Early Understanding of Mind: Origins and Development (pp. 115-132). Hove: L.E.A.

Call, J. \& Tomasello, M. (2008). Does the chimpanzee have a theory of mind? 30 years later. Trends in Cognitive Sciences, 12 (5), 187-192.

CARLSON, S., MOSES, L. \& HIX, H. (1998). The role of inhibitory control in young children's difficulties with deception and false belief. Child Development, 69, 672-691.

CARPENDALE, J. \& LEWIS, CH. (2006). How children develop social understanding. Oxford: Blackwell.

Carpenter, M., Nagell, K. \& Tomasello, M. (1998). Social cognition, joint attention and communicative competence from 9 to 15 months of age. Monographs of the Society for Research in Child Development, 63 (4), serial $\mathrm{n}^{\circ} 255$.

CARPENTER, M. \& TOMASELlo, M. (2000). Joint attention, cultural learning, and language acquisition: implications for children with autism. En A. M. Wetherby \& B. M. Prizant (Eds.), Communication and language issues in autism and pervasive developmental disorder: A transactional developmental perspective (pp. 31-54). Baltimore, MD: Brookes.

Charman, T. (2003). Why is joint attention a pivotal skill in autism? Philosophical Transactions of the Royal Society B, 358, 315-324.

Charman, T., Baron-Cohen, S., Swettenham, J., Baird, G., Cox, A. \& Drew, A. (2000). Testing joint attention, imitation and play as infancy precursors to language and theory of mind. Cognitive Development, 15, 481-498.

Charman, T., Baron-Cohen, S., Swettenham, J., Baird, G., Drew, A. \& CoX, A. (2003). Predicting language outcome in infants with autism and pervasive developmental disorders. International Journal of Language and Communication Disorders, 38, 265-285.

CHEUNG, H., HSUAN-CHIH, C., CRED, N., HG, L., WANG, S. P. \& Mo, L. (2004). Relative roles of general and complementation language in theory-of-mind development: evidence from Cantonese and English. Child Development, 75 (4), 11551170 .

De Rosnay, M. \& Hughes, C. (2006). Conversation and theory of mind: do children talk their way to socio-cognitive understanding? British Journal of Developmental Psychology, 24, 7-37.

De Villiers, J. (2005). Can language acquisition give children a point of view? En J. W. Astington \& J. A. Baird (Eds.), Why language matters for theory of mind (pp. 186-219). Nueva York: Oxford University Press.

De Villiers, J. \& Pyers, J. (2002). Complements to Cognition. A longitudinal study of the relationship between complex syntax and false belief understanding. Cognitive Development 17, 1037-1060.

De Villiers, J. \& De Villiers, P. (2000). Linguistic determinism and the understanding of false beliefs. En P. Mitchell \& K. Riggs (Eds.), Children's Reasoning and the Mind (pp. 191-228). Hove, UK: Psychology Press.

De VilLiers, P. (2005). The role of language in theory of mind development: what deaf children tell us. En J. W. Astington \& J. A. Baird (Eds.), Why language matters for theory of mind (pp. 266-297). NuevaYork: Oxford University Press.

Denham, S., Zoller, D. \& CouCHOUd, E. (1994). Socialization of preschoolers' emotion understanding. Developmental Psychology, $30(6), 928-937$.

DuNN, J. (1988). The beginnings of social understanding. Cambridge, MA: Cambridge University Press.

DunN, J. \& BRown, J. (1993). Early conversations about causality: Content, pragmatics and developmental change. British Journal of Developmental Psychology, 11, 107-123.

DunN, J., BROWN, J., SLOMKOWSKI, C., TeSLA, C. \& YoungBlade, L. (1991). Young children's understanding of other people's feelings and beliefs: individual differences and their antecedents. Child Development, 62, 1352-1366.

ENSOR, R. \& Hughes, C. (2008). Content or connectedness? Mother-child talk and early social understanding. Child Development, 79 (1), 201-216. 
Filippova, E. \& Astington, J. (2008). Further development in social reasoning revealed in discourse irony understanding. Child Development, 79 (1), 126-138.

FISHER, N. (2002). Language and Theory of Mind en children with autism and learning difficulties. Poster presentado en la conferencia internacional "Why language matters for Theory of Mind", Toronto, Canadá.

Gale, E., De Villiers, P., DE Villiers, J. \& Pyers, J. (1996). Language and theory of mind in oral deaf children. En A. Stringfellow, D. Cahana-Amitay, E. Hughes \& A. Zukowski (Eds.), Proceedings of the $20^{\text {th }}$ Annual Boston University Conference on Language Development (Volume 1, pp. 213-224). Somerville, MA: Cascadilla Press.

Hale, C. \& TAger-Flusberg, H. (2003). The influence of language on theory of mind: a training study. Developmental Science, 6(3), 346-359.

HAPPÉ, F. (1993). Communicative competences and theory of mind in autism: A test of relevance theory. Cognition, 48, 101119.

HAPPÉ, F. (1995). The role of age and verbal ability in the theory of mind task performance of subjects with autism. Child Development, 66, 843-855

HARRIS, P. (2005). Conversation, pretense, and Theory of Mind. En J. W. Astington \& J. A. Baird (Eds.), Why language matters for theory of mind (pp. 70-83). Nueva York: Oxford University Press.

JACQUES, S. \& ZELAZO, D. (2005). Language and the development of cognitive flexibility: implications for theory of mind. En J. W. Astington \& J. A. Baird (Eds.), Why language matters for theory of mind (pp. 144-162). Nueva York: Oxford University Press.

Jenkins, J. M. \& Astington, J. W. (1996). Cognitive factors and family structure associated with Theory of Mind development in young children. Developmental Psychology, 32, 70-78.

Karmiloff-Smith, A. (1992). Beyond modularity. Cambridge, MA: Cambridge University Press. [Trad. cast. de J. C. Gómez \& M. Núñez, Más allá de la modularidad. Madrid: Alianza, 1994].

Lagattuta, K. \& Wellman, H. (2002). Differences in early parent-child conversations about negative versus positive emotions: Implications for the development of emotion understanding. Developmental Psychology, 38, 564-580.

LesLIE, A. (1987). Pretense and representation: The origins of Theory of Mind. Psychological Review, 94, 412-426.

Leslie, A. \& Polizzi, P. (1998). Inhibitory processing in the false belief task: two conjetures. Developmental Science, 1, 247253.

Lohmann, H. \& Tomasello, M. (2003). The role of language in the development of false belief understanding: a training study. Child Development, 74 (4), 1130-1144.

Meins, E., FERNYHOUGH, C., RuSSELL, J. \& CLARK-CARTER, D. (1998). Security of attachment as a predictor of symbolic and mentalising abilities: A longitudinal study. Social Development, 7, 1-24.

Meins, E., Fernyhough, C., Wainwright, R., DAS GuPTA, M., FradLey, E. \& TuCKey, M. (2002). Maternal mind-mindedness and attachment security as predictors of theory of mind understanding. Child Development, 73, 1715-1726.

Milligan, K., Astington, J. \& DACK, L. (2007). Language and Theory of Mind: Meta-Analysis of the relation between language ability and false-belief understanding. Child Development, 78 (2), 622-646.

OlsON, D. (1988). On the origins of beliefs and other intencional states in children. En J. W. Astington, P. L. Harris \& D. R. Olson (Eds.), Developing theories of mind (pp. 414-426). Nueva York: Cambridge University Press.

O'NeILL, D. (1996). Two-year-old children's sensitivity to a parent's knowledge state when making requests. Child Development, 67, 659-677.

PeRNER, J. (1988). Higher order beliefs and intentions in children's understanding of social interaction. En J. W. Astington; P. L. Harris \& D. Olson (Eds.), Developing Theories of Mind (pp. 271-292). Cambridge, UK: Cambridge University Press.

Perner, J. (1991). Understanding the representational mind. Cambridge, MA: MIT Press. [Trad. cast. de M. A. Galmarini: Comprender la mente representacional. Barcelona: Paidós, 1994].

Perner, J., RufFman, T. \& LeeKam, S. (1994). Theory of mind is contagious: You catch it from your sibs. Child Development, 65, 1228-1238.

Perner, J., SPRUNG, M., Zauner, P. \& HAider, H. (2003). Want-that is understood well before think-that, say-that, and false belief: A test of deVilliers' linguistic determinism on German speaking children. Child Development, 74, 179-188.

Peterson, C. \& Siegal, M. (1995). Deafness, conversation and the theory of mind. Journal of Child Psychology and Psychiatry, 36, 459-474.

PlAUT, D. \& KARMILOFF-SMith, A. (1993). Representational development and theory-of-mind computations. Behavioral and Brain Sciences, 16, 70-71.

PrEMACK, D. \& WOODRUFF, G. (1978). Does the chimpanzee have a theory of mind? The Behavioral and Brain Sciences, 1 , 515-526.

Rivière, A. \& EsPaÑol, S. (2003). La suspensión como mecanismo de creación semiótica. Estudios de Psicología, 24, 261-275.

Ruffman, T., Slade, L. \& CROWE, E. (2002). The relation between child and mothers' mental state language and theory-ofmind understanding. Child Development, 73, 734-751.

Ruffman, T., Slade, L., Rowlandson, K., Rumsey, C. \& Garnham, A. (2003). How language relates to belief, desire, and emotion understanding. Cognitive Development, 18, 139-158.

Russell, J., MAuthner, N., SHARPE, S. \& TidSWELl, T. (1991). The "Windows task" as a measure of strategic deception in preschoolers' strategic deception. British Journal of Developmental Psychology, 12, 301-314.

SCHICK, B., DE Villiers, P., DE Villiers, J. \& HOFFMEISTER, R. (2007). Language and theory of mind: A study of deaf children. Child Development, 78 (2), 376-396.

SLADE, L. \& RufFMAN, T. (2005). How language does (and does not) relate to theory of mind: A longitudinal study of syntax, semantics, working memory and false belief. British Journal of Developmental Psychology, 23, 1-26.

Slaughter, V., Peterson, C. \& Mackintosh, E. (2007). Mind what mother says: narrative input and theory of mind in typical children and those on the autism spectrum. Child Development, 78 (3), 839-858.

SORCE, J., EmDe, R., CAmpos, J. \& KLinnert, M. (1985). Maternal emotional signaling: its effect on the visual cliff behavior of infants and young children. Journal of Experimental Child Psychology, 35, 369-390.

TAger-Flusberg, H. \& Joseph, R. (2005). How language facilitates the acquisition of false belief understanding in children with autism. En J. W. Astington \& J. A. Baird (Eds.), Why language matters for theory of mind (pp. 298-318). Nueva York: Oxford University Press.

Tager-Flusberg, H. \& Sullivan, K. (1994). Predicting and explaining behavior: a comparison of autistic, mentally retarded and normal children. Journal of Child Psychology and Psychiatry, 35, 1059-1075. 
TARdif, T. \& Wellman, H. (2000). Acquisition of mental state language in Mandarin-and Cantonese-speaking children. Developmental Psychology, 36, 25-43.

TAUMMOEPEAU, M. \& RUFFMAN, T. (2006). Mother and infant talk about mental states relates to desire language and emotion understanding. Child Development, 77 (2), 465-481.

TAumoepeau, M. \& RufFman, T. (2008). Stepping stones to other's minds: maternal talk relates to child mental state language and emotion understanding at 15, 24 and 33 months. Child Development, 79 (2), 284-302.

Tomasello, M. (2001). Perceiving intentions and learning words in the second year of life. En M. Tomasello \& E. Bates (Eds.), Language development: the essential readings (pp. 111-128). Oxford: Blackwell.

Tomasello, M. (2006). Acquiring linguistic constructions. En D. Kuhn \& R. Siegler (Eds.), Handbook of Child Psychology (pp.255-298). Nueva York: Wiley.

Tomasello, M. \& BARton, M. (1994). Learning words in non-ostensive contexts. Developmental Psychology, 30, 639-650.

Tomasello, M. \& CARPENTER, M. (2007). Shared intentionality. Developmental Science, 10(1), 121-125.

Tomasello, M., Carpenter, M. \& LiszKowski, U. (2007). A new look to infant pointing. Child Development, 78 (3), $705-$ 722.

Vygotsky, L. S. (1978). Mind in Society. Cambridge, MA: Harvard University Press.

Wellman, H., Cross, D. \& Watson, J. (2001). Meta-analysis of theory of mind development: The truth about false belief. Child Development, 72, 655-684.

Wellman, H., PhILLIPS, A., DUNPHY-LeliI, S. \& LALONDE, N. (2004). Infant social attention predicts preschool social cognition. Developmental Science, 7 (3), 283-288.

WellmaÑ, H. M. (1993). Early understanding of the mind: the normal case. En S. Baron-Cohen, H. Tager-Flusberg \& D. J. Cohen (Eds.), Understanding Other Minds: perspectives from autism (pp. 10-39). Nueva York: Oxford University Press.

Wimmer, H. \& Perner, J. (1983). Beliefs about beliefs: representation and the constraining function of wrong beliefs in young children's understanding of deception. Cognition, 13, 103-128.

WinNER, E. \& LEEKAN, S. (1991). Distinguishing irony from deception: Understanding the speaker's second-order intention. British Journal of Developmental Psychology, 9, 257-270.

Woolfe, T., WANT, S. \& Siegal, M. (2002). Signposts to development: Theory of mind in deaf children. Child Development, 73, 768-778.

ZeLAZO, P. D. \& Frye, D. (1997). Cognitive complexity and control: A theory of the development of deliberate reasoning and intencional action. En M. Stamenov (Ed.), Language structure, discourse, and the access to consciousness (pp. 113-153). Ámsterdam: John Benjamins. 